\title{
Erratum: Magnetically induced electric polarization in an organometallic magnet
}

[Phys. Rev. B 82, 060402(R) (2010)]

\author{
V. S. Zapf, M. Kenzelmann, F. Wolff-Fabris, F. Balakirev, and Y. Chen
}

Q (Received 28 May 2018; published 11 June 2018)

DOI: 10.1103/PhysRevB.97.219901

The word "organometallic" in the title and the paper should be replaced by "metal-organic." This material is not organometallic since no carbon atom bonds directly to the metal atom. 